\title{
Consumption pattern of gabapentinoids in a tertiary health-care system: a five year study from 2012-2017
}

\author{
Tejus A. ${ }^{1}$, Sharmila Sinha ${ }^{2 *}$
}

${ }^{1}$ Department of Pharmacology, Army College of Medical Sciences, Delhi Cantt, New Delhi, India

${ }^{2}$ Department of Pharmacology, Command Hospital, Lucknow, Uttar Pradesh, India

Received: 29 April 2019

Accepted: 06 May 2019

\section{*Correspondence to:}

Dr. Sharmila Sinha, Email:sherrysinha2002@ yahoo.com

Copyright: (C) the author(s), publisher and licensee Medip Academy. This is an openaccess article distributed under the terms of the Creative Commons Attribution NonCommercial License, which permits unrestricted noncommercial use, distribution, and reproduction in any medium, provided the original work is properly cited.

\begin{abstract}
Background: Treating chronic pain has always been a great challenge for the treating physician. As the available pharmacotherapy do not always yield desired results, wider armamentarium is needed in tackling the chronic pain. Hence gabapentinoids are often considered, but it has led to widespread off label use of these groups of medications. Hence a study of consumption pattern was carried out to assess the usage of gabapentinoids.

Methods: The consumption pattern of gabapentinoids (gabapentin/ pregabalin/ any combination) was recorded from the medical stores and dispensary of tertiary care teaching hospital.

Results: There is an upward trend in the usage of all gabapentinoids between 2012 to 2017 and pregabalin was seen to be the commonly used gabapentinoid. In the recent years apart from individual gabapentinoids there is a trend favoring use of FDCs of gabapentinoids with nortryptyline and methylcobalamine.

Conclusions: The increase in the usage pattern should alarm us to look into the off label uses of gabapentinoids. As there is a possibility of abuse and misuse of gabapentinoids clear guidelines regarding the same is the need of the hour.
\end{abstract}

Keywords: Consumption pattern, Gabapentinoids, Off label uses

\section{INTRODUCTION}

Pain continues to be an old challenge in the medical history and remains the most common reason for seeking the medical attention. ${ }^{1,2} \mathrm{~A}$ consensus definition of pain by the International Association for the study of pain (IASP) in 1986 defines it as "an unpleasant sensory and emotional experience which is primarily associated with tissue damage or described in terms of such damage, or both". 3,4 Pain that persists beyond the normal process of healing is considered to be chronic pain. ${ }^{5,6}$
The eleventh revision of International Classification of Diseases (ICD) of the World Health Organization (WHO) has categorized chronic pain into seven groups namely chronic primary pain, chronic cancer pain, chronic posttraumatic and postsurgical pain, chronic neuropathic pain, chronic headache and orofacial pain, chronic visceral pain, and chronic musculoskeletal pain. ${ }^{5}$ Globally one in five individuals suffer from chronic pain and almost $1 / 5$ th of the visit to a physician is accounted for the same. The prevalence of chronic pain in India has a huge range that varies between $9-41 \% .^{6,7}$ 
A multidisciplinary approach is often resorted for treating chronic pain which includes medical treatment (Pharmacotherapy, Nerve blocks-caudal/ paravertebral/ stellate ganglion/ sympathetic, spinal cord stimulation, surgery-discectomy), physical therapy (Acupuncture, exercise programme, hydrotherapy, massage and transcutaneous electric nerve stimulation), psychological treatments (Biofeedback, education) and group psychotherapy. ${ }^{8}$

Amongst the pharmacotherapy Non-steroidal antiinflammatory drugs (NSAIDs) are commonly used for managing mild to- moderate pain, especially those pain with a component of inflammation. ${ }^{9-12}$ Non-selective NSAIDs by inhibiting cyclooxygenase (COX) enzyme 1 and 2 inhibits prostaglandin production to provide pain relief. They are associated with various adverse effects like dyspepsia, rash, gastro-duodenal ulcers, esophagitis, asthma exacerbations, dizziness, seizures, confusion, stroke and heart failure. ${ }^{12}$ The selective COX-2 inhibitors (Etoricoxib, valdecoxib and celecoxib) offer same efficacy and improved gastric tolerance compared to non-selective COX inhibitors, but result in an increased risk of hypertension, edema, stroke and myocardial infarction due to prostacyclin (PGI2) synthesis inhibition without affecting thromboxane A2 (TXA2) synthesis. ${ }^{12}$

Opioid analgesics usually restricted for providing relief to moderate-severe pain as they are associated with serious adverse effects like constipation, respiratory depression, tolerance, dependence and abuse. ${ }^{11-13}$ The use of prescription opioids always had been a controversial issue with studies suggesting that there has been a dramatic upsurge in volume of opioid use which has triggered a corresponding increase in incidence of addictions and deaths due to overdosages. ${ }^{11}$

Antidepressants inhibiting serotonin and noradrenaline reuptake are useful in treating pain disorders like fibromyalgia, neuropathic pain, rheumatoid conditions, low back pain, headache and are referred as adjuvant analgesics. ${ }^{14}$ Tricyclic antidepressants (TCAs) effectively relieve chronic pain, but their use is associated with frequent adverse effects like drowsiness, dry mouth, urinary retention, constipation, orthostatic hypotension, sedation, memory impairment, weight gain and prolongation of QTc interval due to their actions on multiple receptors. ${ }^{14,15}$

Selective serotonin reuptake inhibitors (SSRIs) like fluoxetine though have shown limited analgesic activity but have better safety profile compared to TCAs. SSRIs, unlike the TCAs, do not cause cardiovascular side effects and seizures, but can cause nausea, diarrhea, increased incidence of epistaxis and ecchymosis, anxiety, insomnia, decreased libido, erectile dysfunction, ejaculatory delay, dullness of intellectual abilities and concentration. ${ }^{11,14-15}$

Various adjuvant analgesics antiepileptics (Phenytoin, oxcarbamazepine, lamotrigine, topiramate, gabapentin, pregablin), antiarrythmics (Lignocaine and mexiletine) and clonidine, an alpha-2-agonist are commonly used for postherpetic neuralgia, trigeminal neuralgia and diabetic neuropathy. ${ }^{16}$ Baclofen and tizanidine, the antispasmodic agents are routinely used against chronic pain. ${ }^{10,16}$

A number of drug classes are often utilized for tackling chronic pain, but all the groups tend to produce some adverse effects. Hence, there is a need to look into new group of drugs. In this scenario, non-opioid, non-NSAID option gabapentinoids - Gabapentin and pregabalin look promising and are often used as a first line option for neuropathic pain. ${ }^{9}$

Gabapentin, a GABA derivatives acts through modulation of $\alpha 2 \delta$ - 1 subunit of the voltage sensitive calcium channels thereby reducing the release of glutamate and lowers the excitability of neurons. It was approved as an adjuvant antiepileptic in 1993. Pregabalin, a newer congener of gabapentin was approved by USFDA in 2004 for postherpetic neuralgia and diabetic neuropathy; later on, in 2007 was approved for fibromyalgia. ${ }^{9}$

Gabapentinoids used off-label for various indications including trigeminal neuralgia, migraine, bipolar disorders, restless leg syndrome and various pain syndromes. Research suggests a raising trend in prescription of gabapentinoids and an estimate suggest upto $95 \%$ prescription of gabapentinoids are for its off label uses. ${ }^{9,17,18}$ There are many question raised regarding off label usage, with even warning about the fatalities and abuse liabilities at overdosages by the databases of various national and international pharmacovigilance centres. ${ }^{17,18}$ Even the promoters of both gabapentinoids have been fined for promoting off label use. ${ }^{9}$

Hence this study was planned to study the usage pattern of gabapentinoids in a busy tertiary care centre, this could demonstrate a raising trend in the use of gabapentinoids.

Aim and objective was to study the consumption pattern of gabapentinoids (Gabapentin and pregabalin) in a tertiary healthcare system.

\section{METHODS}

The consumption pattern of gabapentinoids was assessed in a tertiary care centre and a teaching hospital following an institutionally sponsored delivery of healthcare. After approval from institutional ethics committee, the consumption pattern of all the gabapentinoids namely gabapentin, pregabalin and any of their combinations was assessed over a five-year period from 2012 to 2017. The study duration was selected based on the increase in case studies highlighting the probable abuse potential of gabapentinoids during this period. The study will help to highlight any change in the trends of consumption of gabapentinoids. 
The consumption pattern was obtained using the data from the software installed at the hospital dispensary to check the daily expense from the dispensary of the hospital which issues medicines based on the prescriptions by various outpatient departments. This was counterchecked from the records of stock load received at the medical stores of the hospital. All the doses of gabapentin (100 and $300 \mathrm{mg}$ ), pregabalin (75 and $150 \mathrm{mg}$ ) were treated as single entity to obtain the overall consumption pattern. This was essential to eliminate the bias as the unavailability of one dose of medications could have been countered by the usage of another dose.

During the course of the study it was noticed that few combination of gabapentinoids were also being utilised. Hence the combination of either gabapentin or pregabalin with any other medication (e.g.: Nortryptyline/ methylcobalamine) were also considered to demonstrate a correct usage pattern of gabapentinoids.

\section{RESULTS}

The study clearly demonstrates the increase in consumption of gabapentinoids during the entire study duration. Pregabalin was the most preferred gabapentinoid in our study followed by gabapentin, with the combinations pregabalin+methylcobalamine and pregabalin+ nortryptyline were only used in 2017 . The consumption of gabapentinoids almost tripled from 2012 to 2017 with Pregabalin being the most preferred gabapentinoid (Table 1, Figure 3). There is a steady increase in the consumption of gabapentin and pregabalin from 2012 to 2016 followed by a steep spike in 2017 (Figure 1, Figure 2 and Figure 3).

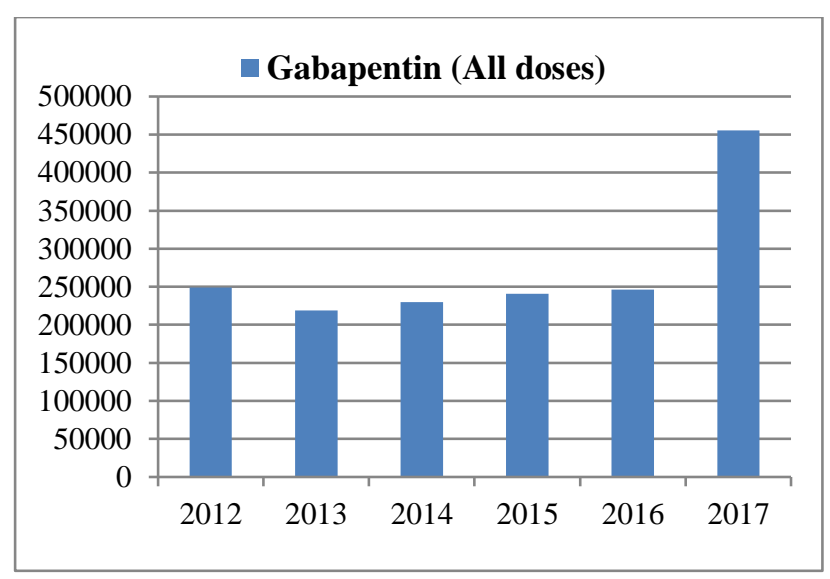

Figure 1: Consumption pattern of Gabapentin.

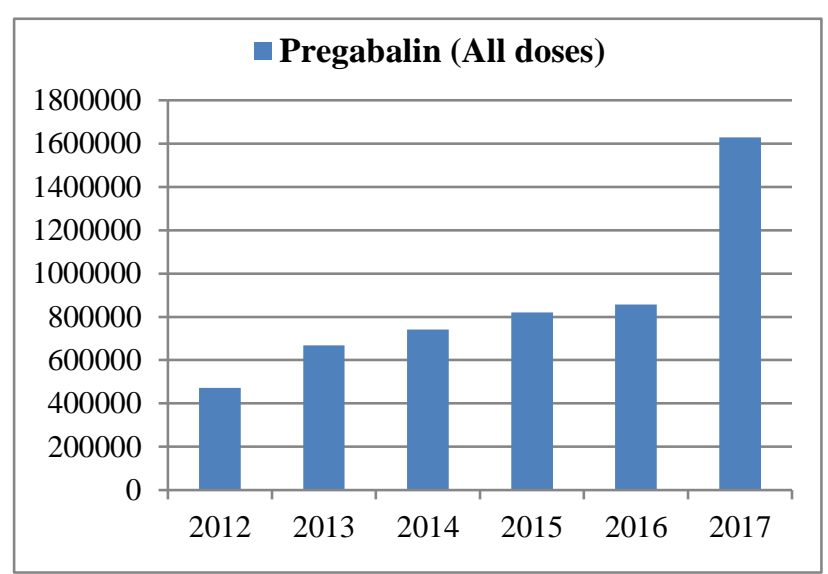

Figure 2: Consumption pattern of Pregabalin.

Table 1: Consumption pattern of gabapentinoids.

\begin{tabular}{|lllll|}
\hline & Gabapentin & Pregabalin & Pregabalin + Methylcobalamine & Pregabalin + Nortryptyline \\
\hline 2012 & $249000(34.54 \%)$ & $472000(65.46 \%)$ & - & - \\
\hline 2013 & $219000(24.69 \%)$ & $668250(75.31 \%)$ & - & - \\
\hline 2014 & $230000(23.66 \%)$ & $742250(76.34 \%)$ & - & - \\
\hline 2015 & $240600(22.69 \%)$ & $820000(77.31 \%)$ & - & - \\
\hline 2016 & $246000(22.33 \%)$ & $856000(77.67 \%)$ & - & - \\
\hline 2017 & $455190(21.84 \%)$ & $1628307(78.15 \%)$ & $8000(0.003 \%)$ & $3000(0.001 \%)$ \\
\hline
\end{tabular}

In 2012, the percentage of gabapentin use was at its highest at $34.54 \%$ of all the gabapentinoids prescribed but was lower than that of pregabalin at $65.46 \%$. The use of gabapentin was shown to decline gradually with the usage percentage reaching as low as $21.84 \%$ of total gabapentinoids prescribed in 2017. On the other hand, pregabalin accounting for $65.46 \%$ of all the prescribed gabapentinoids in 2012 then shows a progressive increase year on year to reach its highest usage percentage of $78.15 \%$ of the prescribed gabapentinoids by 2017 (Table
1, Figure 4). Every year the volume of use of gabapentinoids is shown to increase but in 2017 along with increased volume, newercombinations (Pregabalin+methylcobalamine/ Nortryptyline) make their appearance in the formulary of the hospital.

\section{DISCUSSION}

The current study was an effort to bring out the overall pattern of consumption of gabapentinoids in a tertiary care 
centre. Our study clearly highlights pregabalin to be the most commonly used gabapentinoid. In line with results of our study, Mishra R et al (2016) compared the postoperative analgesia potential of gabapentin and pregabalin in those undergoing laparoscopic cholecystectomy. They demonstrated that in comparison to gabapentin, pregabalin group had lower visual analogue scale score, less use of rescue analgesics and opioids, concluding pregabalin to be a better analgesic compared to gabapentin. ${ }^{19}$

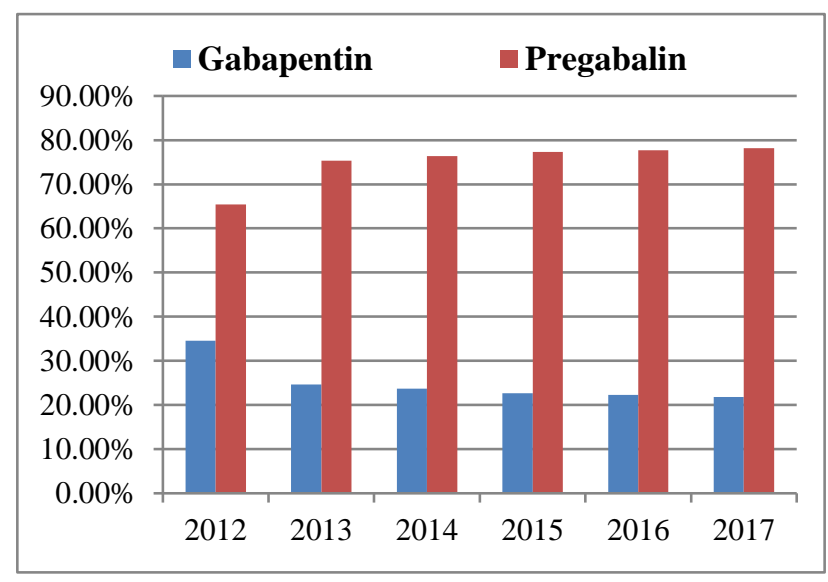

Figure 3: Comparison of percentage in consumption pattern of Gabapentin and Pregabalin.

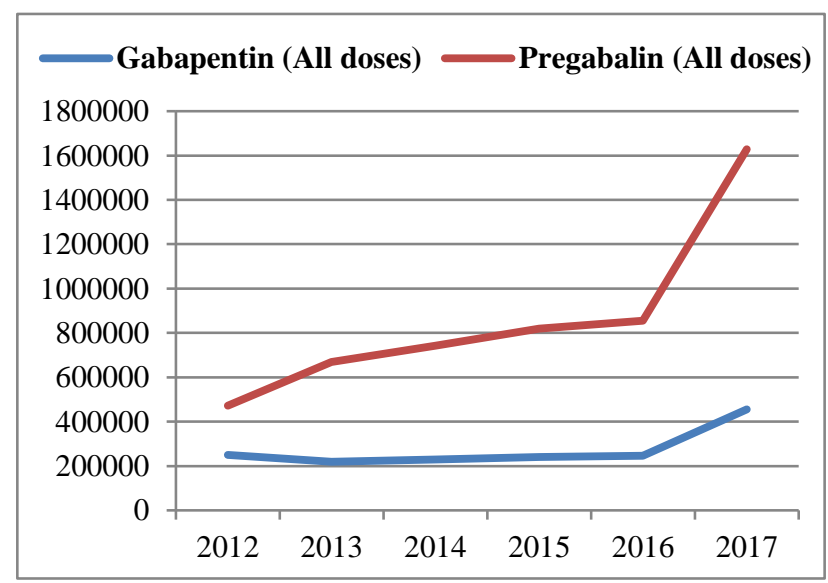

Figure 4: Comparison of consumption pattern of Gabapentin and Pregabalin.

There has been nearly a three times increase in consumption of gabapentinoids over a period from 2012 to 2017 in our study. This again correlates with the results of Johansen ME (2018), who analysed the usage pattern of gabapentinoids in United States between 2002 to 2015, which showed nearly a thrice increase in gabapentinoid use. ${ }^{20}$ Goodman CW et al (2017), in their perspective on usage pattern of gabapentinoids reported that 64 million gabapentin dispensed in 2016, nearly a twice increase from 39 million dispensed in $2012 .^{8}$

Johansen ME (2018), also noted that the usage of gabapentin showed a statistically significant increase after
2008 and usage of pregabalin plateaued by $2008 .^{20}$ This is in contrast to our results where we could see a sharp spike in the usage of both gabapentin and pregabalin in 2017. The reasons for the same is difficult to determine, probably a sharp increase in the off-label usage.

In our study pregabalin was found to be the most commonly prescribed gabapentinoid. This is in stark contrast to the findings of Johansen ME (2018), who reported gabapentin to be the most commonly used gabapentinoid in United States from 2002 to 2015. ${ }^{20}$ As pregabalin availability is restricted in United states as it is a controlled substance probably could be the reason for lesser usage. ${ }^{21}$ In India, there is no such restriction on pregabalin usage and few pharmacological advantages with pregabalin like higher bioavailability of more than $90 \%$, shorter half-life $(6.3 \mathrm{hrs})$ and rapid renal elimination without metabolism producing a predictable response appears to be the suitable explanation for its preference over gabapentin. ${ }^{22}$

In our study another significant finding is the usage of fixed dose combination (FDC) of pregabalin with methylcobalamine and nortryptyline. Vasudevan D et al (2014), in their pilot study (MAINTAIN) compared the efficacy and safety of the FDC of methylcobalamine and pregabalin to monotherapy of pregabalin for neuropathic pain. ${ }^{23}$ The study demonstrated that combination provides pain relief, improves sleep and nerve function. However still larger trials are warranted before the FDC could be officially added to the formulary for treating neuropathic pain. The FDC of pregabalin and antidepressant (Nortryptyline $10 \mathrm{mg}$ ) has been opined to improve compliance and lowers side effects. The expert opinion of Saxena AK et al (2018) on the combination appears it to be a synergistic combination which can be useful to improve the compliance. ${ }^{24}$

Increased utilization of gabapentinoids for off-label uses remains a concern, but off late there are evidence surfacing with potential misuse and abuse potential of gabapentinoids. Evoy KE et al (2017) in their systematic review supported the concern and suggested about $1.6 \%$ prevalence of abuse of gabapentinoids in general population, but also suggested that the prevalence to be as high as $3-68 \%$ amongst opioid abusers. ${ }^{25}$ Smith RV et al (2016) in their systematic review have highlighted the reason for the same. ${ }^{26}$ Gomes $\mathrm{T}$ et al (2017) demonstrated that co-prescribing opioids with gabapentinoids increases the risk of deaths due to respiratory depression. ${ }^{27}$

The drawback of our study is it determines only the consumption pattern, which does not clearly highlight the indication of use of gabapentinoids. A proper prescription analysis of gabapentinoids will bring out the same.

\section{CONCLUSION}

The gabapentinoids are being used extensively for various varieties of chronic pain, fuelling the off-label use of the 
same. The pattern appears alarming with potential of misuse/ abuse being associated with the same. The prescription pattern of gabapentinoids needs a comprehensive revision, with suitable guidelines by the international regulators to influence the practicing physicians. Further studies assessing the prescription patterns and devising a method to detect abuse liabilities is the need of the hour.

\section{Funding: No funding sources}

Conflict of interest: None declared

Ethical approval: The study was approved by the Institutional Ethics Committee

\section{REFERENCES}

1. Alles SR, Smith PA. Etiology and Pharmacology of Neuropathic Pain. Pharmacol Rev. 2018;70(2):31547.

2. Raffaeli W, Arnaudo E. Pain as a disease: an overview. J Pain Res. 2017;10:2003-8. Published 2017 Aug 21.

3. Andreu V, Arruebo M. Current progress and challenges of nanoparticle based therapeutics in pain management. J Control Release. 2018 Jan 10;269:189213.

4. Orr PM, Shank BC, Black AC. The Role of Pain Classification Systems in Pain Management. Crit Care Nurs Clin N Am. 2017;29(4):407-18.

5. Treede RD, Rief W, Barke A, Aziz Q, Bennett MI, Benoliel R, et al. A classification of chronic pain for ICD-11. Pain. 2015 Jun;156(6):1003-7.

6. Dureja GP, Jain PN, Shetty N, Mandal SP, Prabhoo R, Joshi M, et al. Prevalence of chronic pain, impact on daily life, and treatment practices in India. Pain Pract. 2014 Feb;14(2):E51-62.

7. Walters JL, Baxter K, Chapman H, Jackson $\mathrm{T}$, Sethuramachandran A, Couldridge M, et al. Chronic Pain and Associated Factors in India and Nepal: A Pilot Study of the Vanderbilt Global Pain Survey. Anesth Analg. 2017;125(5):1616-26.

8. Fashler SR, Cooper LK, Oosenbrug ED, Burns LC, Razavi S, Goldberg L, et al. Systematic Review of Multidisciplinary Chronic Pain Treatment Facilities. Pain Res Manag. 2016;2016:5960987.

9. Goodman CW, Brett AS. Gabapentin and Pregabalin for Pain-Is Increased Prescribing a Cause for Concern? N Engl J Med. 2017;377(5):411-4.

10. Joshi GM. Holistic care in chronic pain. Indian J Pain. 2017;31(2):77-9.

11. Rosenblatt RA, Catlin M. Opioids for Chronic Pain: First Do No Harm. Ann Fam Med. 2012;10(4):300-1.

12. Crofford LJ. Use of NSAIDs in treating patients with arthritis. Arthritis Res Ther. 2013;15(Suppl 3):2-10.

13. Yaksh TL, Wallace MS. Opioids, Analgesia, and Pain Management. In: Brunton LL, Chabner BA, Knollmann BC, editors. Goodman and Gilman's The Pharmacological Basis of Therapeutics. 12th ed. San Diego, California: McGraw-Hill; 2011:481-527.
14. Dharmshaktu P, Tayal V, Kalra BS. Efficacy of Antidepressants as Analgesics: A Review. J Clin Pharmacol. 2012;52(1):6-17.

15. Gupta N, Case AA. Adjuvant analgesics. In: Smith HS, Pilitsis JG, editors. The Art and Science of Palliative Medicine. $1^{\text {st }}$ Ed. New York: AME Publishing Company; 2014:209-222.

16. Dureja GP, Iyer RN, Das G, Ahdal J, Narang P. Evidence and consensus recommendations for the pharmacological management of pain in India. J Pain Res. 2017;10:709-36.

17. Wallach JD, Ross JS. Gabapentin Approvals, OffLabel Use, and Lessons for Post-marketing Evaluation Efforts. JAMA. 2018;319(8):776-8.

18. Morrison EE, Sandilands EA, Webb DJ. Gabapentin and pregabalin: do the benefits outweigh the harms? J R Coll Physicians Edinb. 2017 Dec;47(4):310-3.

19. Mishra R, Tripathi M, Chandola HC. Comparative clinical study of gabapentin and pregabalin for postoperative analgesia in laparoscopic cholecystectomy. Anesth Essays Res. 2016;10(2):2016.

20. Johansen ME. Gabapentinoid Use in the United States 2002 Through 2015. JAMA Intern Med. 2018;178(2):292-4.

21. Wise J. Gabapentinoids should not be used for chronic low back pain, meta-analysis concludes. BMJ. 2017;358:j3870.

22. Parsons G. Guide to the management of gabapentinoid misuse. Therapy focus prescriber. 2018;1:25-8.

23. Vasudevan D, Naik MM, Mukaddam QI. Efficacy and safety of methylcobalamin, alpha lipoic acid and pregabalin combination versus pregabalin monotherapy in improving pain and nerve conduction velocity in type 2 diabetes associated impaired peripheral neuropathic condition. [MAINTAIN]: Results of a pilot study. Ann Indian Acad Neurol. 2014;17(1):19-24.

24. Saxena AK, Jain P, Dureja GP, Venkitachalam A, Goswami S, Usmani $\mathrm{H}$, et al. Pharmacological management of neuropathic pain in India: A consensus statement from Indian experts. Indian $\mathrm{J}$ Pain. 2018;32(3):132-44.

25. Evoy KE, Morrison MD, Saklad SR. Abuse and Misuse of Pregabalin and Gabapentin. Drugs. 2017 Mar; 77(4):403-26.

26. Smith RV, Havens JR, Walsh SL. Gabapentin misuse, abuse, and diversion: A systematic review. Addiction (Abingdon, England). 2016;111(7):1160-74.

27. Gomes T, Juurlink DN, Antoniou T, Mamdani MM, Paterson JM, van den Brink W. Gabapentin, opioids, and the risk of opioid-related death: A populationbased nested case-control study. PLoS Med. 2017;14(10):e1002396.

Cite this article as: Tejus A, Sinha S. Consumption pattern of gabapentinoids in a tertiary health-care system: a five year study from 2012-2017. Int J Basic Clin Pharmacol 2019;8:1233-7. 